\title{
Enhancement of potency and stability of human extracellular superoxide dismutase
}

\author{
Sunghwan Kim ${ }^{1,2}$, Hae-Young Kim ${ }^{1}$, Jung-Ho Kim ${ }^{1}$, Jung-Hye Choi ${ }^{1}$, Won-Kook Ham ${ }^{1}$, Yoon-Jae Jeon ${ }^{1}$, \\ Hara $\mathrm{Kang}^{3}$ \& Tae-Yoon Kim ${ }^{1, *}$ \\ ${ }^{1}$ Department of Dermatology, College of Medicine, The Catholic University of Korea, Seoul 137-040, ${ }^{2}$ New Drug Development Center, \\ Daegu-Gyungpook Medical Innovation Foundation, Daegu 701-310, ${ }^{3}$ Division of Life Science, College of Life Science and Bioengineering, \\ Incheon National University, Incheon 406-772, Korea
}

Cells express several antioxidant enzymes to scavenge reactive oxygen species (ROS) responsible for oxidative damages and various human diseases. Therefore, antioxidant enzymes are considered biomedicine candidates. Among them, extracellular superoxide dismutase (SOD3) had showed prominent efficacy against asthma and inflammation. Despite its advantages as a biomedicine, the difficulty in obtaining large quantity of active recombinant human SOD3 (rhSOD3) has limited its clinical applications. We found that a significant fraction of overexpressed rhSOD3 was composed of the inactive apo-enzyme and its potency against inflammation depended on the rate of metal incorporation. Also, purified rhSOD3 was unstable and lost its activity very quickly. Here, we suggest an ideal preparative method to express, purify, and store highly active rhSOD3. The enzymatic activity of rhSOD3 was maximized by incorporating metal ions into rhSOD3 after purification. Also, albumin or polyethylene glycol prevented rapid inactivation or degradation of hSOD3 during preparative procedures and long-term storage. [BMB Reports 2015; 48(2): 91-96]

\section{INTRODUCTION}

Reactive oxygen species (ROS), such as hydrogen peroxide and superoxide radicals, are generated either by enzymatic reactions that transfer electrons in the mitochondria and the endoplasmic reticulum, or by external stimuli such as irradiation. ROS can induce cellular oxidative damages by modifying proteins and nucleic acids. Therefore, cells have developed diverse systems to reduce ROS. First, ROS-generating systems

${ }^{*}$ Corresponding author. Tel: +82-2-2258-6221; Fax: +82-2-34828261; E-mail: tykimder@catholic.ac.kr

http://dx.doi.org/10.5483/BMBRep.2015.48.2.093

Received 2 May 2014, Revised 17 May 2014, Accepted 23 May 2014

Keywords: Extracellular superoxide dismutase, Inflammation, Metalloenzyme, Reactive oxygen species, Refolding are tightly regulated, so as not to produce high levels of ROS. Second, cells have antioxidant systems to scavenge ROS, including small scavengers such as glutathione, vitamin $\mathrm{C}$, and vitamin $\mathrm{E}$, in addition to antioxidant enzymes, such as superoxide dismutase (SOD), catalase, and peroxidase.

Despite cellular regulatory systems, ROS can accumulate upon prolonged stimulation. It has been shown that ROS is closely related to numerous chronic human diseases, including inflammation $(1,2)$. Therefore, many antioxidant molecules and cellular antioxidant enzymes have been considered as medicines (3-6). Among them, SOD scavenges superoxide radicals, which directly modify proteins or generate its derivatives, such as $\mathrm{H}_{2} \mathrm{O}_{2}$ and $\mathrm{ONOO}^{-}$(7-9). Mammalian cells express SOD1, SOD2, and SOD3 in cytosol, mitochondria, and the extracellular region, respectively (9). Mice lacking SOD showed organ failure or increased sensitivity to oxygen toxicity and inflammatory responses and exogenous SOD has alleviated inflammatory responses (10-15), suggesting that SOD family members are critical for preventing ROS-derived diseases.

SOD3 is a strong biomedicine candidate. First, exogenous SOD3 can accomplish its functions without cell penetration, since it is originally an extracellular anti-oxidant enzyme. Second, an earlier study suggested that SOD3 also inhibited inflammatory responses via non-enzymatic functions (13), providing additional impetus for the use of SOD3. Third, transgenic mice over-expressing SOD3 displayed no abnormal phenotypes. Instead, they were more resistant to inflammatory responses $(10,16,17)$, while SOD1 transgenic mice showed neuronal abnormalities (18). Last, SOD3 exhibited a longer half-life $(\sim 20 \mathrm{~h})$ in the blood, whereas SOD1 had a very short half-life ( $20 \mathrm{~min})$, possibly due to its rapid renal clearance $(19,20)$.

One obstacle to developing recombinant human SOD3 (rhSOD3) into a novel biomedicine is the high costs for producing large quantities of active protein. Despite many efforts, rhSOD3 expressed from bacteria or yeast has showed very low activity compared to rhSOD3 from mammalian cells (Table 1), possibly due to lack of proper folding and maturation machineries. Therefore, mammalian cells are the most suitable

ISSN: 1976-670X (electronic edition)

Copyright (C) 2015 by the The Korean Society for Biochemistry and Molecular Biology

(c) This is an open-access article distributed under the terms of the Creative Commons Attribution Non-Commercial License (http://creativecommons.org/licenses/by-nc/3.0) which permits unrestricted non-commercial use, distribution, and reproduction in any medium, provided the original work is properly cited. 
Preparation of potent and stable human SOD3

Sunghwan Kim, et al.

Table 1. Activities of recombinant human SOD3

\begin{tabular}{|c|c|c|c|c|c|}
\hline Protein & Host & Prep method & Unit/mg & Source & Reference \\
\hline SOD3 & Bacteria & $\begin{array}{l}\text { Ni column } \\
\text { Gel filtration }\end{array}$ & $50-510$ & Inclusion body & $(34,36)$ \\
\hline SOD3 & Bacteria & $\begin{array}{l}\text { Ni column } \\
\text { Heparin column }\end{array}$ & 120 & Soluble lysate & (29) \\
\hline SOD3 & Yeast & Not purified & 760 & Culture media & (35) \\
\hline SOD3 & $\begin{array}{l}\text { Mammalian } \\
\mathrm{CHO}\end{array}$ & $\begin{array}{l}\text { Antibody affinity } \\
\text { lon exchange } \\
\text { Heparin column }\end{array}$ & $>50,000$ & Culture media & (33) \\
\hline SOD3 & $\begin{array}{l}\text { Mammalian } \\
\text { 293T-EBNA }\end{array}$ & $\begin{array}{l}\text { Ni column* } \\
\text { Suggested method** }\end{array}$ & $\begin{array}{l}30,000 \\
60,000\end{array}$ & Culture media & In this study \\
\hline C195S & $\begin{array}{l}\text { Mammalian } \\
\text { 293T-EBNA }\end{array}$ & Suggested method** & 80,000 & Culture media & In this study (no data) \\
\hline C219S & $\begin{array}{l}\text { Mammalian } \\
\text { 293T-EBNA }\end{array}$ & Suggested method** & 140,000 & Culture media & In this study (no data) \\
\hline 209E & $\begin{array}{l}\text { Mammalian } \\
\text { 293T-EBNA }\end{array}$ & Suggested method** & 120,000 & Culture media & In this study (no data) \\
\hline
\end{tabular}

The enzyme activity was determined after nickel purification $(*)$ or after incubating purified rhSOD3 with $0.1 \% \mathrm{BSA}$ and $100 \mu \mathrm{M} \mathrm{CuSO}_{4} / \mathrm{ZnCl} 2$ for $24 \mathrm{hrs}(* *)$.

over-expression system for producing active rhSOD3. SOD3 is a secretory protein that matures in the ER and Golgi apparatus and undergoes post-translational modifications, including glycosylation, disulfides, and proteolysis. In addition, a proteolytic variant, 209E, which is missing the C-terminal heparin-binding domain, can be observed in normal tissue (21-23). Considering its diverse post-translational modifications, the difficulty in producing rhSOD3 using a bacterial system is explainable.

During the course of this study, we noticed that even the rhSOD3 expressed from mammalian cells were not fully active. Moreover, purified rhSOD3 lost its activity very quickly. The activity of rhSOD3 was maximized by incorporating metal ions post-translationally and rhSOD3 was stabilized by serum albumin (BSA) or polyethylene glycol (PEG). This study will provide invaluable information for the preparation of potent rhSOD3 in advance of preclinical or clinical applications.

\section{RESULTS AND DISCUSSION}

\section{Purification of rhSOD3}

rhSOD3 tagged with C-terminal $\mathrm{His}_{6}$ was purified directly from culture media by single-step purification on a nickel column (Fig. 1A). Purified rhSOD3 showed the correct monomer size, around $27 \mathrm{kDa}$ and half of the rhSOD3 formed a dimer with an intermolecular disulfide bond, whereas recombinant 209E showed no dimeric bands in non-reducing SDS-PAGE (Fig. 1B), as noticed elsewhere $(21,22)$.

\section{Catalytic activity of rhSOD3}

SOD3 is a metalloenzyme that uses copper and zinc ions as cofactors for catalysis. If the basal amount of metal ions in the culture media is insufficient to accommodate over-expressed
A

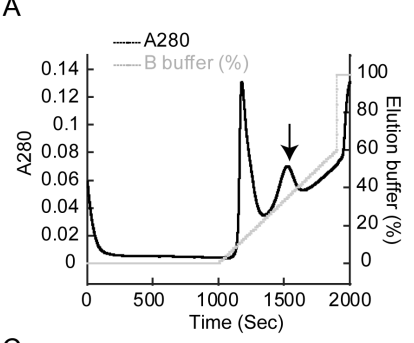

B

C

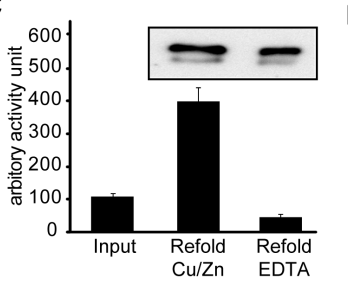

$\mathrm{D}$

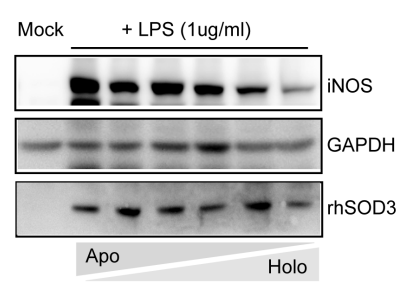

Fig. 1. Purification of rhSOD3 and post-translational metal incorporation. (A) Culture media containing rhSOD3 were filtered and loaded onto a nickel column, followed by washing and gradient elution. Arrow indicates the peak corresponding fraction of pure rhSOD3. (B) Elution fractions of rhSOD3 and recombinant 209E were analyzed by non-reducing and reducing SDS- PAGE with blue staining. (C) Generation of holo- and apo-enzyme from purified rhSOD3. Purified rhSOD3 corresponding to 100 units of the initial activity were denatured and refolded in the presence of either $\mathrm{Cu} / \mathrm{Zn}$ or EDTA. Enzyme activities and protein amounts after refolding were compared (Western blot analysis in the enclosed figure). (D) Anti-inflammatory effect by enzymatically active rhSOD3. Prevention of iNOS induction by LPS correlated to percentage of holo-enzyme.

rhSOD3, metal-free rhSOD3 can be secreted into the media. Cells employ the copper chaperones CCS1 and ATOX1 to assist with copper incorporation into SOD1 and SOD3, re- 
spectively $(24,25)$. However, SOD3 has been shown to bind metal cofactors under in vitro conditions without chaperone proteins (24). Therefore, we examined if exogenous copper/zinc ions could enhance the catalytic activity of purified rhSOD3. Right after elution from the nickel column, rhSOD3 was dialyzed into either PBS or PBS containing $50 \mu \mathrm{M} \mathrm{Cu} / \mathrm{Zn}$ ions, followed by additional dialysis into PBS to remove the unbound metal ions. rhSOD3 dialyzed into the buffer containing $\mathrm{Cu} / \mathrm{Zn}$ ions was much more active compared to when dialyzed into plain PBS (data not shown). This result suggested that some over-expressed rhSOD3 was secreted from cells without metal incorporation or the rhSOD3 lost metal ions after secretion, resulting in the formation of apo-enzyme.

In order to estimate the approximate percentage of apo-enzyme in the purified sample, we denatured purified rhSOD3 and allowed it to refold in the presence of either a metal chelator, EDTA, to maximize the apo-enzyme percentage or in the presence of $\mathrm{Cu} / \mathrm{Zn}$ ions to maximize the holo-enzyme percentage. Refolded rhSOD3 in the presence of $\mathrm{Cu} / \mathrm{Zn}$ ions showed nearly 4-fold higher activity compared to the initial activity of purified rhSOD3 (Fig. 1C). However, refolded rhSOD3 in the presence of EDTA showed the least activity. rhSOD3 did not show any visible aggregation during refolding in any condition (data not shown) and protein amounts were quite similar in both conditions (Fig. 1C enclosed figure). These results suggested that around $75 \%$ of the purified rhSOD3 was apo-enzyme or unfolded enzyme which can recover its activity by post-translational metal incorporation and refolding.

Anti-inflammatory efficacy of rhSOD3 is correlated to activity Although rhSOD3 demonstrated non-enzymatic activity against asthma (12), its enzymatic activity must be critical for anti-inflammation considering the impact of ROS on inflammation. In order to examine the anti-inflammatory effect of rhSOD3 depending on the rate of metal incorporation, Raw 264.7 cells activated by LPS were treated with different ratios
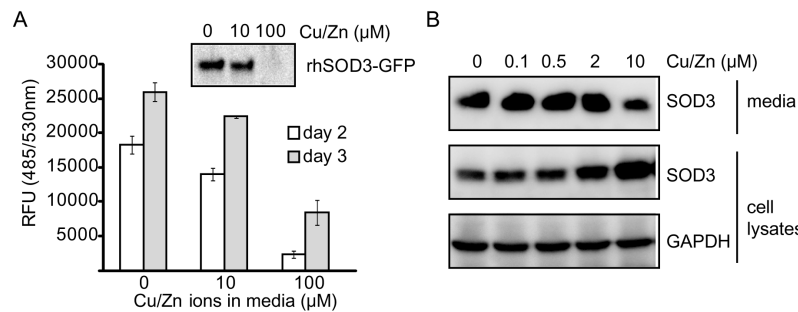

Fig. 2. Supplementation with metal ions decreased secretion of rhSOD3 from cells. 293T cells stably expressing rhSOD3 or rhSOD3-EGFP were cultured in DMEM and supplemented with various concentration of $\mathrm{CuSO}_{4} / \mathrm{ZnCl}_{2}$ for 3 days. (A) GFP fluorescence signals in the 293T-rhSOD3-EGFP culture media were measured. Amounts of rhSOD3-EGFP in culture media were compared by Western blot analysis (enclosed). (B) Amounts of intracellular and extracellular rhSOD3 in 293T-rhSOD3 cells were compared by Western blot analysis. of holo-enzyme to apo-enzyme. LPS-activated cells showed significant induction of iNOS, one of the inflammatory markers (26). The inhibitory efficacy of rhSOD3 on iNOS induction decreased as the percentage of holo-enzyme was reduced (Fig. 1D). This correlation between the enzyme activity of rhSOD3 and its efficacy emphasizes the importance of preparing catalytically active rhSOD3 for future applications.

\section{Effects of metal ions on rhSOD3 expression from cells}

We noticed that over-expressed SOD3 was secreted without metal ions, suggesting that the amount of metal ions available was insufficient. Therefore, we also investigated whether supplementation of $\mathrm{Cu} / \mathrm{Zn}$ ions increased the expression level of active rhSOD3. Unexpectedly, exogenous supplementation of $\mathrm{Cu} / \mathrm{Zn}$ ions significantly reduced the secretion of rhSOD3. The extracellular fluorescence signal of rhSOD3-GFP decreased with a higher concentration of $\mathrm{Cu} / \mathrm{Zn}$ ions in the media and Western blot analysis also supported this result (Fig. 2A and
$A$

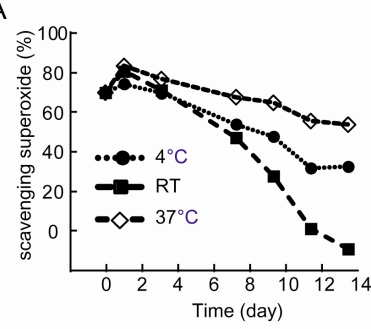

C

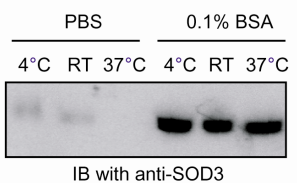

D

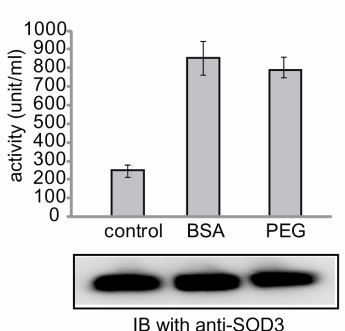

B

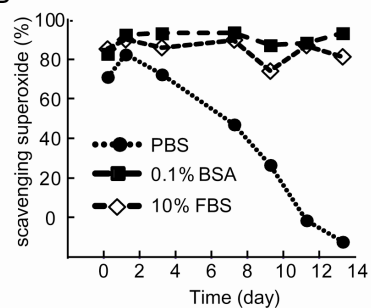

$E$

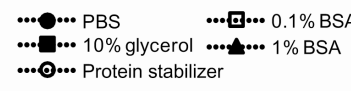

- 60

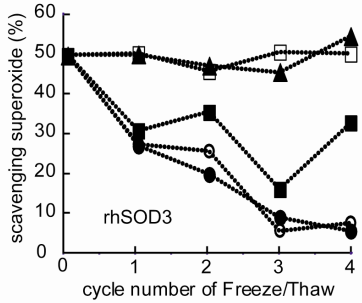

Fig. 3. Stabilization of rhSOD3. (A) Purified rhSOD3 lost its catalytic activity over time. The catalytic activity of rhSOD3 stored in PBS at different temperatures was monitored for 13 days. (B) The catalytic activity of rhSOD3 stored in PBS, $0.1 \% \mathrm{BSA}$, or $10 \%$ FBS at $37^{\circ} \mathrm{C}$ is shown. (C) After 13 days of incubation, the amount of rhSOD3 was analyzed by Western blotting. (D) Freshly purified rhSOD3 were dialyzed under three different conditions; dialysis into PBS buffer, dialysis into PBS buffer after mixing $0.1 \%$ BSA with purified rhSOD3, and dialysis into PBS buffer containing $0.1 \%$ PEG. (E) Purified rhSOD3 was stored in PBS, $0.1 \%$ BSA, $1 \%$ BSA, $10 \%$ glycerol, or a protein stabilizing cocktail, and their catalytic activities were measured after repeated freeze/thaw cycles. 
2B). Instead, rhSOD3 seemed to accumulate inside cells that had higher metal concentrations (Fig. 2B), suggesting that $\mathrm{Cu} / \mathrm{Zn}$ ions might inhibit secretion or induce internalization of SOD3. Therefore, we concluded that supplementation of $\mathrm{Cu} / \mathrm{Zn}$ ions in culture media was unsuitable for enhancing the expression of active rhSOD3.

\section{Stabilization of active rhSOD3}

We noticed that rhSOD3 was very unstable even after optimal purification procedures. Purified rhSOD3 lost almost half of its initial activity in PBS buffer at $37^{\circ} \mathrm{C}$ within 7 days (Fig. 3A). Activity loss was also detected at low temperatures. Therefore, it was necessary to optimize the preparation and storage conditions to stabilize active rhSOD3. First, purified rhSOD3 was supplemented with $0.1 \%$ BSA or $10 \%$ FBS. Both $0.1 \%$ BSA and $10 \%$ FBS maintained the catalytic activity of rhSOD3 for up to 13 days at $37^{\circ} \mathrm{C}$ (Fig. $3 \mathrm{~B}$ ), suggesting that $\mathrm{BSA}$ is sufficient to stabilize rhSOD3. Previous studies showed that $\mathrm{Cu} / \mathrm{Zn}$ SOD can be fragmented, following copper release and/or non-enzymatic glycation $(27,28)$. Purified rhSOD3 disappeared almost completely after 13 days in PBS buffer (Fig. 3C), but $0.1 \%$ BSA prevented this disappearance. This result suggested that rhSOD3 loses its activity due to fragmentation or degradation.

Second, we examined whether rhSOD3 loses its activity during the purification procedure, which is performed at low temperature over 2 days. Right after elution from the affinity column, rhSOD3 was either mixed with $0.1 \%$ BSA, followed by dialysis into PBS, or it was dialyzed directly into PBS containing $0.1 \%$ PEG, another known protein stabilizer. Interestingly, rhSOD3 prepared with $0.1 \%$ BSA or $0.1 \%$ PEG showed much higher activity than with PBS only (Fig. 3D). Protein amount was not affected by the different dialysis methods (Fig. 3D enclosed figure), suggesting that BSA (or PEG) can also prevent the release of metal ions from purified rhSOD3.

Finally, the effects of freeze/thaw cycles on rhSOD3 were investigated. In general, repeated freezing/thawing is known to destabilize proteins. Freshly purified rhSOD3 were frozen in liquid nitrogen and stored at $-80^{\circ} \mathrm{C}$ within different buffer conditions. After thawing, the catalytic activity of rhSOD3 was immediately measured. A single freeze/thaw cycle reduced rhSOD3 activity by approximately $50 \%$ in PBS. However, BSA $(1 \%$ and $0.1 \%)$ completely prevented rhSOD3 activity loss from the freeze/thaw cycle (Fig. 3E). 10\% glycerol also partially stabilized rhSOD3 activity. However, a commercially available protein stabilizing cocktail (Thermo Fisher) failed to stabilize active rhSOD3.

\section{DISCUSSION}

In this study, we found that exogenous copper and zinc ions can maximize rhSOD3 activity post-translation and that BSA or PEG can stabilize active rhSOD3 either by protecting against fragmentation or the release of metal ions. However, rhSOD3 purified from bacteria did not show a significant increase in catalytic activity in the presence of exogenous $\mathrm{Cu} / \mathrm{Zn}$ ions (data not shown), suggesting that the native-like conformations must be important for the chaperone free post-translational insertion of $\mathrm{Cu} / \mathrm{Zn}$ ions into rhSOD3.

The heparin-binding domain of SOD3 is cleaved naturally, resulting in $209 \mathrm{E}(20,29)$. Therefore, we investigated whether the heparin-binding domain affected rhSOD3 stability. However, recombinant 209E lost its activity similarly to rhSOD3 and was also protected by BSA or PEG during long term storage and a freeze/thaw cycle (data not shown). These results indicate that the heparin-binding domain contributes neither to the stability nor the activity loss of rhSOD3. Instead, it may regulate the plasma levels of rhSOD3 by interacting with the cell surface or the extracellular matrix $(20,29)$.

In this study, we optimized the preparative method for active rhSOD3. After purification from mammalian cell culture media, rhSOD3 was combined with $0.1 \%$ BSA (or $0.1 \%$ PEG) and $50 \mu \mathrm{M} \mathrm{Cu} / \mathrm{Zn}$ ions to maximize and stabilize its catalytic activity, and then free $\mathrm{Cu} / \mathrm{Zn}$ ions were removed by dialysis for future applications. The enhanced catalytic activity of rhSOD3 prepared using the above procedure was well maintained even after removing free $\mathrm{Cu} / \mathrm{Zn}$ ions (data not shown). 209E and two cysteine mutants, C195S and C219S, prepared through the above procedure showed similar or better activity than wild-type rhSOD3 (Table 1). Although C195S is thought to be a hyperactive mutant due to a deficiency of the inhibitory disulfide bond (C107-C195) (30), its activity was only 1.33 -fold higher than wild-type rhSOD3. Compared to rhSOD3 purified from bacteria or yeast, rhSOD3 prepared using our procedure displayed 100- to 1,000-fold higher activity $(25,31-34)$ (Table 1). The optimized procedure recommended in this study can help to overcome practical obstacles in the development of rhSOD3 as a novel biomedicine.

\section{MATERIALS AND METHODS}

\section{Cloning and mammalian cell culture}

The full length human SOD3 and 209E variant, from Met1 to Glu227, containing a C-terminal His6 tag or an enhanced green fluorescence protein (EGFP) tag was inserted into pcDNA3.1 (Invitrogen) using HindIII and EcoRI or HindIII and Xbal, respectively. Plasmids encoding hSOD3 and 209E variants were transfected into 293T-EBNA cells with Attractene (Qiagen) based on the manufacturer's instructions. One day after transfection, the media were replaced with serum-free Dulbecco's Modified Eagle Medium (DMEM). 293T-EBNA cells stably expressing rhSOD3-EGFP were selected using G418 (Invitrogen) and enriched by fluorescence-activated cell sorting (FACS). 293T-EBNA and Raw264.7 cells were maintained with DMEM containing 10\% FBS.

\section{Protein expression and purification}

Five days after transfection, culture media containing rhSOD3 were collected, filtrated, and loaded onto HiTrap Chelating HP 
column (GE Healthcare). After loading, the column was washed with more than 50 column volumes of washing buffer, 50 $\mathrm{mM} \mathrm{NaPO}_{4}, 500 \mathrm{mM} \mathrm{NaCl}$, and $30 \mathrm{mM}$ imidazole. Then, rhSOD3 and 209E were eluted by increasing the elution buffer containing $500 \mathrm{mM}$ imidazole (Fig. 1A), followed by dialysis in PBS or the indicated buffer conditions. The concentration of purified rhSOD3 was determined based on a BSA standard curve with a protein assay dye (Bio-Rad).

\section{Activity assay for SOD}

To measure the enzymatic activity of rhSOD3, the rate of superoxide radical formation was quantified spectrophotometrically. A $20 \mu \mathrm{l}$ sample was mixed with $200 \mu \mathrm{l}$ of 200 $\mu \mathrm{M}$ xanthine (Sigma) and $50 \mu \mathrm{M}$ WST-1 (Dojindo) in PBS. After adding 0.0005 unit XOD (Sigma), the increase in the formazan dye signal was immediately recorded using a colorimetric method at $\mathrm{A}_{450}$. The generation of a formazan dye signal was determined kinetically, and absolute SOD activity was determined from the dilution factor exhibiting $50 \%$ inhibition $\left(\mathrm{IC}_{50}\right)$ on the inhibition curve.

\section{Anti-inflammatory effects of rhSOD3}

At $70 \%$ confluence, Raw 264.7 cells were starved with serum-free DMEM for $6 \mathrm{~h}$ prior to treatment with $1 \mu \mathrm{g} / \mathrm{ml} \mathrm{lip-}$ opolysaccharide (LPS). rhSOD3 was added to cells with different ratios of holo-enzyme to apo-enzyme, but a constant amount corresponding to 100 units $/ \mathrm{ml}$ concentration of $100 \%$ holo-enzyme. Cells were harvested after $24 \mathrm{~h}$ incubation by directly adding SDS sample buffer containing protease inhibitors. iNOS, GAPDH, and hSOD3 were analyzed by Western blot analysis with anti-NOS2 (Santa Cruz Biotechnology), anti-GAPDH (Santa Cruz Biotechnology), and anti-hSOD3 (AbCam) antibodies.

\section{Monitoring activity loss of rhSOD3}

Purified rhSOD3 was incubated in different conditions such as $0.1 \% \mathrm{BSA}$, or $10 \%$ fetal bovine serum (FBS) at $4^{\circ} \mathrm{C}$, room temperature (RT), or $37^{\circ} \mathrm{C}$. The activity of $10 \mu$ purified rhSOD3 corresponding to 4 units of the initial activity was monitored for 13 days. To assess the effects of freeze/thaw cycles on activity loss, purified rhSOD3 in PBS supplemented with $1 \%$ BSA, $0.1 \%$ BSA, $10 \%$ glycerol, or protein stabilizing cocktail (Thermo Scientific) was quickly frozen with liquid nitrogen. After thawing, the activity of $2 \mu \mathrm{l}$ rhSOD3 corresponding to 1 units initial activity was determined.

\section{Supplementation of copper and zinc ions and refolding} In order to check the effects of $\mathrm{Cu} / \mathrm{Zn}$ on expression of SOD3, 0,10 , and $100 \mu \mathrm{M} \mathrm{CuSO}_{4} / \mathrm{ZnCl}_{2}$ mixtures were added to the culture media used for 293T cells stably expressing rhSOD3. One day later, the amount of expressed and secreted rhSOD3 (-EGFP) in the culture media was determined by Western blotting with anti-SOD3 antibody or GFP fluorescence. To assess the effects of exogenous $\mathrm{Cu} / \mathrm{Zn}$ on purified rhSOD3, $50 \mu \mathrm{M}$
$\mathrm{CuSO}_{4} / \mathrm{ZnCl}_{2}$ was either directly combined with purified rhSOD3 or purified rhSOD3 was dialyzed into PBS containing $10 \mu \mathrm{M} \mathrm{CuSO} / \mathrm{ZnCl}_{2}$, followed by the removal of free $\mathrm{Cu} / \mathrm{Zn}$. For refolding, rhSOD3 was denatured using $6 \mathrm{M}$ Guanidine $\mathrm{HCl}$ and refolded by dialysis into PBS with either $10 \mu \mathrm{M}$ $\mathrm{CuSO}_{4} / \mathrm{ZnCl}_{2}$ or $10 \mathrm{mM}$ EDTA.

\section{ACKNOWLEDGEMENTS}

This research was supported by the Basic Science Research Program through the National Research Foundation of Korea (NRF) funded by the Ministry of Education, Science, and Technology (NRF-2013R1A1A2008027) and the Bio \& Medical Technology Development Program of the National Research Foundation (NRF) funded by the Ministry of Science, ICT \& Future Planning (NRF-2013M3A9A3050567). We give many thanks to the Integrative Research Support Center of the Catholic University of Korea for FACS.

\section{REFERENCES}

1. Finkel T (2005) Radical medicine: treating ageing to cure disease. Nat Rev Mol Cell Biol 6, 971-976

2. Rosanna DP and Salvatore C (2012) Reactive oxygen species, inflammation, and lung diseases. Curr Pharm Des 18, 3889-3900

3. Niwa $Y(1989)$ Lipid peroxides and superoxide dismutase (SOD) induction in skin inflammatory diseases, and treatment with SOD preparations. Dermatologica 179 Suppl 1, 101-106

4. Kim BH, Na KM, Oh I et al (2013) Kurarinone regulates immune responses through regulation of the JAK/STAT and TCR-mediated signaling pathways. Biochem Pharmacol 85, 1134-1144

5. Sunitha K, Hemshekhar M, Thushara RM et al (2013) $\mathrm{N}$-Acetylcysteine amide: a derivative to fulfill the promises of N-Acetylcysteine. Free Radic Res 47, 357-367

6. Traber MG and Stevens JF (2011) Vitamins C and E: beneficial effects from a mechanistic perspective. Free Radic Biol Med 51, 1000-1013

7. Yamakura F and Kawasaki H (2010) Post-translational modifications of superoxide dismutase. Biochim Biophys Acta 1804, 318-325

8. Fridovich I (1995) Superoxide radical and superoxide dismutases. Annu Rev Biochem 64, 97-112

9. Zelko IN, Mariani TJ and Folz RJ (2002) Superoxide dismutase multigene family: a comparison of the CuZn-SOD (SOD1), Mn-SOD (SOD2), and EC-SOD (SOD3) gene structures, evolution, and expression. Free Radic Biol Med 33, 337-349

10. Lee YS, Cheon IS, Kim BH, Kwon MJ, Lee HW and Kim TY (2013) Loss of Extracellular Superoxide Dismutase Induces Severe IL-23-Mediated Skin Inflammation in Mice. J Invest Dermatol 133, 732-741

11. Asikainen TM, Huang TT, Taskinen E et al (2002) Increased sensitivity of homozygous Sod2 mutant mice to oxygen toxicity. Free Radic Biol Med 32, 175-186 
12. Kwon MJ, Jeon YJ, Lee KY and Kim TY (2012) Superoxide dismutase 3 controls adaptive immune responses and contributes to the inhibition of ovalbumin-induced allergic airway inflammation in mice. Antioxid Redox Signal 17, 1376-1392

13. Kwon MJ, Han J, Kim BH, Lee YS and Kim TY (2012) Superoxide dismutase 3 suppresses hyaluronic acid fragments mediated skin inflammation by inhibition of toll-like receptor 4 signaling pathway: superoxide dismutase 3 inhibits reactive oxygen species-induced trafficking of toll-like receptor 4 to lipid rafts. Antioxid Redox Signal 16, 297-313

14. Tominaga $T$, Hachiya $M$, Shibata $T$, Sakamoto $Y$, Taki $K$ and Akashi M (2012) Exogenously-added copper/zinc superoxide dismutase rescues damage of endothelial cells from lethal irradiation. J Clin Biochem Nutr 50, 78-83

15. Kim DW, Hwang HS, Kim DS et al (2011) Effect of silk fibroin peptide derived from silkworm Bombyx mori on the anti-inflammatory effect of Tat-SOD in a mice edema model. BMB Rep 44, 787-792

16. Na K, Kim KE, Park ST and Kim, T. Y. (2007) EC-SOD suppresses contact hypersensitivity in mouse skin by impairing Langerhans cell migration. J Invest Dermatol 127, 1930-1937

17. Kim Y, Kim BH, Lee $\mathrm{H}$ et al (2011) Regulation of skin inflammation and angiogenesis by EC-SOD via HIF-1alpha and NF-kappaB pathways. Free Radic Biol Med 51, 1985-1995

18. Jaarsma D, Haasdijk ED, Grashorn JA et al (2000) Human $\mathrm{Cu} / \mathrm{Zn}$ superoxide dismutase (SOD1) overexpression in mice causes mitochondrial vacuolization, axonal degeneration, and premature motoneuron death and accelerates motoneuron disease in mice expressing a familial amyotrophic lateral sclerosis mutant SOD1. Neurobiol Dis 7, 623-643

19. Uematsu T, Nagashima S, Umemura K, Kanamaru M and Nakashima M (1994) Pharmacokinetics and safety of intravenous recombinant human superoxide dismutase (NK341) in healthy subjects. Int J Clin Pharmacol Ther 32, 638-641

20. Karlsson K, Sandstrom J, Edlund A, Edlund T and Marklund SL (1993) Pharmacokinetics of extracellular-superoxide dismutase in the vascular system. Free Radic Biol Med 14, 185-190

21. Bowler RP, Nicks M, Olsen DA et al (2002) Furin proteolytically processes the heparin-binding region of extracellular superoxide dismutase. J Biol Chem 277, 16505-16511

22. Enghild JJ, Thogersen IB, Oury TD, Valnickova Z, Hojrup $P$ and Crapo JD (1999) The heparin-binding domain of extracellular superoxide dismutase is proteolytically proc- essed intracellularly during biosynthesis. J Biol Chem 274, 14818-14822

23. Petersen SV, Thogersen IB, Valnickova Z et al (2010) The concentration of extracellular superoxide dismutase in plasma is maintained by LRP-mediated endocytosis. Free Radic Biol Med 49, 894-899

24. Jeney V, Itoh S, Wendt M et al (2005) Role of antioxidant-1 in extracellular superoxide dismutase function and expression. Circ Res 96, 723-729

25. Ahl IM, Lindberg MJ and Tibell LA (2004) Coexpression of yeast copper chaperone (yCCS) and $\mathrm{CuZn}$-superoxide dismutases in Escherichia coli yields protein with high copper contents. Protein Expr Purif 37, 311-319

26. Kim JI, Jang HS and Park KM (2010) Endotoxin-induced renal tolerance against ischemia and reperfusion injury is removed by iNOS, but not eNOS, gene-deletion. BMB Rep 43, 629-634

27. Adachi T, Ohta $H$, Hayashi K, Hirano $K$ and Marklund SL (1992) The site of nonenzymic glycation of human extracellular-superoxide dismutase in vitro. Free Radic Biol Med 13, 205-210

28. Arai K, Maguchi S, Fujii S, Ishibashi H, Oikawa K and Taniguchi N (1987) Glycation and inactivation of human $\mathrm{Cu}-\mathrm{Zn}$-superoxide dismutase. Identification of the in vitro glycated sites. J Biol Chem 262, 16969-16972

29. Petersen SV, Olsen DA, Kenney JM et al (2005) The high concentration of Arg213- -> Gly extracellular superoxide dismutase (EC-SOD) in plasma is caused by a reduction of both heparin and collagen affinities. Biochem J 385, 427-432

30. Petersen SV, Oury TD, Valnickova Z et al (2003) The dual nature of human extracellular superoxide dismutase: one sequence and two structures. Proc Natl Acad Sci U S A $100,13875-13880$

31. Tibell L, Hjalmarsson K, Edlund T, Skogman G, Engstrom A and Marklund SL (1987) Expression of human extracellular superoxide dismutase in Chinese hamster ovary cells and characterization of the product. Proc Natl Acad Sci U S A 84, 6634-6638

32. Son YJ, Bae JY, Chong SH et al (2010) Expression, high cell density culture and purification of recombinant EC-SOD in Escherichia coli. Appl Biochem Biotechnol 162, 1585-1598

33. Chen HL, Yen CC, Tsai TC et al (2006) Production and characterization of human extracellular superoxide dismutase in the methylotrophic yeast Pichia pastoris. J Agric Food Chem 54, 8041-8047

34. Bae JY, Koo BK, Ryu HB et al (2013) Cu/Zn incorporation during purification of soluble human EC-SOD from E. coli stabilizes proper disulfide bond formation. Appl Biochem Biotechnol 169, 1633-1647 